\title{
O TRATAMENTO DA MORFOLOGIA NÃO- CONCATENATIVA PELA MORFOLOGIA DISTRIBUÍDA: \\ O CASO DOS BLENDS FONOLÓGICOS
}

\author{
A distributed morphology account for non- \\ concatenative morphology: the case of phonological \\ blends
}

\author{
Vitor Augusto Nóbrega* \\ Rafael Dias Minussi**
}

\begin{abstract}
RESUMO
Neste artigo, discutimos alguns aspectos da morfologia não-concatenativa do português brasileiro, concentrando-nos nas propriedades morfossintáticas e semânticas dos blends. Tratamos, mais especificamente, dos blends fonológicos, nos quais há a sobreposição de segmentos idênticos das palavras-fontes que os constituem (e.g., roubodizio < roubo + rodizio). Demonstramos que, mesmo nos blends formados através da sobreposição de segmentos fonológicos idênticos, o input imediato para a mesclagem decorre de fatores semânticos e não fonológicos, sendo esses últimos epifenomenais, tal como sugerido por Minussi e Nóbrega (2014).
\end{abstract}

Palavras-chave: morfologia não-concatenativa; blends fonológicos; Morfologia Distribuída.

* Doutorando do Programa de Pós-Graduação em Semiótica e Linguística Geral da Universidade de São Paulo (USP) e membro dos Grupos de Pesquisa: GREMD (Grupo de Estudos em Morfologia Distribuída - USP) e InFoLiC (Investigações (In)formais em Lingua(gem) e Cognição - UNIFESP). Agradeço ao Conselho Nacional de Desenvolvimento Científico e Tecnológico (CNPq) pelo financiamento concedido à minha pesquisa de doutorado da qual o presente artigo é parte (processo 160605/2014-8).

** Professor do Departamento de Letras da Universidade Federal de São Paulo (UNIFESP). Membro dos Grupos de Pesquisa: GREMD (Grupo de Estudos em Morfologia Distribuída - USP) e InFoLiC (Investigações (In)formais em Lingua(gem) e Cognição - UNIFESP). 


\begin{abstract}
In this article, we discuss some aspects of non-concatenative morphology in Brazilian Portuguese, concentrating on the morphosyntactic and semantic properties of blended words. We analyze, more specifically, phonological blends, that is, blends in which identical phonological segments from the source-words are overlapped (e.g., roubodizio 'unfair all-you-can-eat' < roubo 'stealing' + rodizio 'all-you-can-eat'). We demonstrate that even in this sort of blending the immediate input for their formation comes from semantic factors, not phonological ones, being the latter epiphenomenal, as argued for by Minussi and Nóbrega (2014).
\end{abstract}

Keywords: non-concatenative morphology; phonological blends; Distributed Morphology.

\title{
1. INTRODUÇÃo
}

Neste artigo, avançamos a investigação sobre os fenômenos da morfologia não-concatenativa, em especial os blends, em um modelo realizacional não-lexicalista de gramática, a Morfologia Distribuída (doravante, MD; HALLE; MARANTZ, 1993; MARANTZ, 1997). Nosso objetivo principal é endossar a proposta lançada por Minussi e Nóbrega (2014), de que os blends emergem pós-sintaticamente no componente morfológico da gramática através de um $\uparrow$ semântico desencadeado pela Enciclopédia (ou Lista 3).

Em nossa discussão atual, fornecemos evidências adicionais para tal assunção a partir dos chamados blends fonológicos, ou seja, blends em que um ou mais segmentos idênticos - fonemas ou clusters de fonemas - são sobrepostos, tal como ocorre no blend Dilmãe $<$ Dilma + mãe. Em nossa visão, a mesclagem das palavras-fontes que compõem o blend não é motivada por uma eventual semelhança fonológica entre seus segmentos, mas por suas propriedades semânticas. Assim sendo, argumentamos que a sobreposição de segmentos idênticos, quando ocorre, advém de fatores secundários, epifenomenais, paralelamente às assunções de Bye e Svenonius (2012).

Os eixos temáticos que serão explorados neste artigo são: do ponto de vista descritivo, (i) os efeitos estilísticos (e.g., interpretações cômicas ou jocosas) presentes nas palavras formadas através de um processo não-concatenativo, em particular, o blending no português brasileiro (doravante, $\mathrm{PB}$ ); e do ponto de vista explicativo, (ii) a influência do conhecimento enciclopédico nos processos de formação de palavras, juntamente com (iii) o contraste 
entre o conteúdo presente nas raízes - primitivos sintáticos assumidos pela MD - com o conhecimento enciclopédico armazenado na Lista 3.

A discussão está organizada do seguinte modo: na seção 2, caracterizamos a morfologia não-concatenativa e seus processos de formação de palavras, a fim de descrever o blending em meio a esses processos. Na seção 3, recuperamos a classificação dos blends sugerida por Minussi e Nóbrega (2014), com o intuito de apontar as propriedades atribuídas aos blends fonológicos em oposição aos blends morfológicos e semânticos. Na seção 4 , apresentamos a concepção de gramática adotada, bem como os impasses que esses dados colocam para sua organização. Na seção 5 , descrevemos a formação de um blend fonológico, desde a derivação de suas palavras-fontes no componente sintático, até à sua mesclagem no componente morfológico. Por fim, trazemos as considerações finais sobre o artigo.

\section{MoRfologia NÃo-CONCATENATIVA: CARACTERIZAÇÃo E FENÔMENOS REPRESENTATIVOS}

Morfologia não-concatenativa é um rótulo amplo que engloba um conjunto de processos morfológicos variados. Tais processos podem ser agrupados sob esse rótulo pelo fato de que a realização de seus morfemas não se dá, exclusivamente, através da anexação de segmentos fonológicos na borda de uma determinada base. Devido à amplitude e à variedade dos processos morfológicos que apresentam tal característica, encontramos diferentes estratégias para se definir a morfologia não-concatenativa presente nas línguas naturais.

Gonçalves (2009), por exemplo, admite que processos não-concatenativos são aqueles em que a sucessão linear dos morfemas de uma palavra pode ser rompida, de modo que uma informação morfológica não necessariamente se inicia no ponto em que outra termina ${ }^{1}$. Kurisu (2001) considera casos de morfologia não-concatenativa aqueles em que o expoente fonológico de um morfema não pode ser demarcado na sequência de segmentos que compõem uma palavra. Bye e Svenonius (2012), por sua vez, apontam que padrões não-concatenativos são identificados negativamente, ou seja, um fenômeno pode ser considerado um tipo de morfologia não-concatenativa quando fere ideais concatenativos. Os ideais concatenativos mencionados pelos autores assinalam um conjunto de propriedades associadas a uma concepção geral de morfema, a saber:

1 A informação morfológica aludida pelo autor está intrinsecamente associada à forma, ou seja, à realização fonológica de um morfema, e não à informação morfossintática que ele realiza (e.g., traços de pessoa ou número). 
(1) Ideais concatenativos (BYE; SVENONIUS, 2012, p. 429-430)

\section{a. Precedência}

Morfemas são linearmente ordenados (i.e., não sobrepostos);

b. Contiguidade

Morfemas são contíguos (i.e., não descontínuos);

c. Aditividade

Morfemas são adicionados (i.e., não subtraídos);

\section{d. Preservação dos morfemas}

Um morfema é preservado quando demais morfemas são adicionados a ele;

\section{e. Autonomia segmental}

O conteúdo segmental de um morfema é livre de contexto (i.e., morfemas não deveriam ter seu conteúdo segmental determinado por uma entrada lexical ou por outro morfema);

\section{f. Disjunção}

Morfemas estão dissociados um do outro (e.g., não há haplologia).

Com base nesses ideais concatenativos, que regulam a identificação de um morfema prototípico em termos fonológicos, uma série de fenômenos linguísticos pode ser incluída sob o rótulo "morfologia não-concatenativa", por exemplo: casos de afixação segmental (i.e., adição de traços distintivos ou tons a uma base), infixação, reduplicação, morfologia subtrativa, morfologia templática, haplologia, entre outros. Para se caracterizar o blending, em particular, o ideal concatenativo em (1c) - aditividade - é o mais relevante, pois esse processo morfológico envolve a subtração de segmentos fonológicos, levando-se em consideração que a sobreposição de segmentos de suas palavras-fontes caracterizam um tipo de subtração.

Com o intuito de sinalizar as diferentes características presentes nesse tipo de morfologia, Davis e Tsujimura (2014) estabelecem uma tipologia dos processos não-concatenativos, distribuindo-os em dois grupos principais: (i) morfologia não-concatenativa templática e (ii) morfologia não-concatenativa atemplática. A morfologia não-concatenativa templática é encontrada nos fenômenos em que há uma restrição morfológica na forma das bases. Isso é o que acontece de modo abrangente nas línguas semíticas, as quais apresentam uma morfologia não-concatenativa baseada em raízes tri-consonantais - ou seja, formas prosódicas invariantes - e padrões vocálicos ${ }^{2}$ que se "entrelaçam" a elas, flexionando-as ou derivando novas

2 Os padrões vocálicos são combinações de vogais que preenchem lacunas entre as consoantes das raízes em determinados padrões templáticos. Além disso, esses padrões podem apresentar prefixos em sua composição, e são responsáveis, entre outras coisas, por fornecer informação 
palavras. Exemplos são as formas verbais da raiz $\sqrt{ } \mathrm{ktb}$ do árabe, em (2), e o paradigma derivacional da raiz $\sqrt{ }$ šmn, do hebraico, em (3):

(2) Formais verbais do árabe - "escrever"

Verbo

a. katab

b. kattab

c. kaatab
Padrão templático

$\mathrm{CaCaC}$

$\mathrm{CaCCaC}$

$\mathrm{CaaCaC}$
Glosa

'escreveu'

'ditado (particípio passado)'

'correspondido'

(DAVIS; TSUJIMURA, 2014, p. 194)

(3) Paradigma derivacional do hebraico - "gordura"

Palavra derivada

a. šamen

b. šemen

c. šamenet

d. šuman

e. šaman

f. šimen

g. hišmin
Padrão templático

$\mathrm{CaCeC}(\mathrm{ADJ})$

$\mathrm{CeCeC}(\mathrm{N})$

CaCeCet $(\mathrm{N})$

$\mathrm{CuCaC}(\mathrm{N})$

$\mathrm{CaCaC}(\mathrm{V})$

$\mathrm{CiCeC}(\mathrm{V})$

hicCiC (V)
Glosa

gordo

óleo

creme

gordura

engordar

lubrificar/engraxar

engordar

(ARAD, 2005, p. 16)

A morfologia não-concatenativa atemplática, por sua vez, caracteriza fenômenos em que a realização de um determinado morfema não apresenta um conteúdo fonêmico consistente, podendo ser aumentativa, subtrativa ou envolver afixação autossegmental. Um caso de morfologia não-concatenativa aumentativa, por exemplo, são os dados de ênfase adjetival no japonês de Shizuoka (província da região central do Japão). Nessa variedade do japonês, há a adição de uma unidade moraica à base através da inserção de uma consoante ou de uma vogal a fim de se marcar a ênfase (e.g., (i) ozoi 'terrível' $\rightarrow$ onzoi; (ii) katai 'difícil' $\rightarrow$ kattai; (iii) suppai 'azedo' $\rightarrow$ suuppai, cf. DAVIS; TSUJIMURA, 2014, p. 208).

De modo geral, podemos dizer que os processos morfológicos representativos da morfologia não-concatenativa atemplática são os casos de modificação da base, assim caracterizados por Haspelmath e Sims (2010, p. 36). Basicamente, os autores mostram que diversos fenômenos fonológicos afetam um determinado segmento da base, dando origem a uma nova palavra em diversas línguas, por exemplo: a palatalização, o enfraquecimento de consoantes, a geminação, o alongamento de vogais, o encurtamento de

categorial às raízes (cf. BAT-EL, 2001; ARAD, 2005; MINUSSI, 2012).

Revista Letras, Curitiba, N. 91 p. 158-177, JAN./JUN. 2015.

ISSN 2236-0999 (VERSÃo ELETRÔNICA) 
vogais, o vozeamento, a metátese, entre outros. Além desses fenômenos, há também a subtração de segmentos, em que um ou mais segmentos da base são apagados, tal como é possível verificar na formação do plural em murle, no exemplo (4).

$\begin{array}{ll}\text { Singular } & \text { Plural } \\ \text { nyoon } & \text { nyoo } \\ \text { wawoc } & \text { wawo } \\ \text { onyiit } & \text { onyii } \\ \text { rottin } & \text { rotti }\end{array}$

'cordeiro(s)'

(Murle)

(ARENSEN, 1982, p. 40-1 apud HASPELMATH; SIMS, 2010, p. 37)

Haspelmath e Sims (2010) também relacionam a mudança de acento e a mudança de tom como exemplos de uma morfologia não-concatenativa atemplática. A mudança de acento, por exemplo, é frequentemente mencionada no debate sobre os pares verbo-nome de palavras dissilábicas do inglês, tal como conflíct (V) 'conflitar'/cónflict (N) 'conflito' e rebél (V) 'rebelar-se'/rébel (N) 'rebelde'. Esses dados, amplamente discutidos em Kiparsky (1982), mostram que uma alteração na posição do acento, ou seja, na parte autossegmental da palavra, pode dar origem a um novo item lexical.

Finalmente, um terceiro processo de morfologia não-concatenativa atemplática é a conversão ou derivação zero. Nesse processo, não temos a adição ou subtração de um segmento, muito menos algum tipo de afixação autossegmental. O que se verifica é a manutenção integral da forma da base na geração de outro item lexical. Por exemplo, no holandês, há um conjunto de nomes que contém correspondentes verbais fonologicamente idênticos, tal como se verifica em (5). A derivação desses verbos, por sua vez, não envolve a adição ou modificação de nenhum segmento fonológico da base nominal.

\begin{tabular}{clll} 
Nome & \multicolumn{3}{c}{ Verbo } \\
feest & 'festa' & feest & 'fazer uma festa' \\
kalf & 'novilho' & kalver & 'parir um novilho' \\
kwijl & 'baba' & kwijl 'babar'
\end{tabular}

(Holandês)

(DON, 2005, p. 7)

A partir desse breve panorama sobre a distribuição da morfologia não-concatenativa nas línguas naturais, podemos caracterizar o blending como um processo morfológico não-concatenativo atemplático, em que parte dos segmentos de suas palavras-fontes são sobrepostos ou apagados - tal 
como ocorre nos blends fonológicos (e.g., matel $<$ mat $\left.(o)+m(o) t e l^{3}\right)-$, ou simplesmente apagados, sem envolver qualquer tipo de sobreposição, tal como ocorre nos blends morfológicos (i.e., aqueles em que não há sobreposição de segmentos, e ambas as palavras-fontes são truncadas, e.g. cariúcho $<$ cari(oca) + (gaú)cho) e nos blends semânticos (i.e., aqueles em que há uma reanálise semântica de um conjunto de segmentos fonológicos de uma das palavras-fontes, e.g. $\sqrt{ } b o a<\sqrt{ } m a ́ \leftarrow(m a)$ drasta).

A partir dessa caracterização, três questões cruciais emergem sobre os blends, a saber: (i) qual a motivação imediata para a subtração de segmentos na gramática?; (ii) a subtração de segmentos para a formação de um blend decorre da mesma motivação para subtração de segmentos na formação do plural em murle, exemplificado em (4)? Além disso, tendo em mente que a MD é um modelo realizacional de gramática, ou seja, que os expoentes fonológicos dos terminais sintáticos que compõem a palavra são inseridos tardiamente no ramo de $\mathrm{PF}$, (iii) em que momento da derivação de uma palavra a subtração de segmentos ocorre? Iremos sugerir uma resposta para essas perguntas nas próximas seções.

\subsection{A REDUÇÃo DE SEgmentos E A EMERGÊNCIA DE EFEITOS ESTILÍsticos}

Nas línguas românicas, é possível dizer que a morfologia não-concatenativa circunscreve-se à redução ou apagamento de segmentos, sejam nos casos de truncamento de palavras simples ou compostas, exemplificados em (6) e (7), ou nos blends, exemplificados em (8) e (9)4. Um dos propósitos dessa subtração de segmentos, em geral, é a formação de palavras com um determinado efeito estilístico.
a. curta
b. flagra
$<$ curta-metragem
c. refri
$<$ flagrante
d. brincs
$<$ refrigerante
$<$ brincadeira

(SCHER, 2011, p. 62)
a. corto
b. anarco
c. pelu
d. mates

$<$ cortometraje

$<$ anarquista

$<$ peluqueria

$<$ matemática

(Espanhol)

(VÁZQUEZ, 2008 apud SCHER, 2011, p. 62)

3 O sobescrito (“_”) indica o segmento comum que permitiu a sobreposição e os parênteses (“( )”) indicam os segmentos que foram apagados.

4 Insere-se nesse grupo também a hipocorização, ou seja, processo morfológico pelo qual antropônimos são encurtados afetivamente, resultando numa forma truncada que mantém identidade com o nome ou sobrenome original, tal como em Léo < Leonardo (GONÇALVES, 2004a). 
(8)
a. matel
$<$ mato + motel
b. bótimo
$<$ bom + ótimo
c. cantriz
$<$ cantora + atriz
d. impastor
$<$ impostor + pastor
a. suciedad < sucia 'suja' + sociedad 'sociedade' (Espanhol)
b. analfabestia < analfabeta 'analfabeta' + bestia 'besta'
c. burrocracia < burro 'burro' + burocracia 'burocracia'
d. kakaína < cocaína 'cocaína' + kaka 'ruim'

(PIÑEROS, 2000, p. 3-4)

O efeito estilístico aludido diz respeito à interpretação informal atrelada aos dados. Gonçalves (2005), Villalva (2008) e Scher (2011, 2013) mostram que, nos dados de truncamento do português, há uma leitura apreciativa, a qual difere, em grande medida, da interpretação presente na forma plena da palavra (e.g., padaria vs. padoca; cf. SCHER, 2013). Nos blends, por outro lado, a leitura não é essencialmente apreciativa, mas carrega um tom cômico ou jocoso, tal como apontado por Sandmann (1990). A emergência desse significado é um traço característico das línguas românicas no que concerne à subtração de segmentos ${ }^{5}$.

De um ponto de vista translinguístico, entretanto, a redução de segmentos pode estar associada a diversos fenômenos gramaticais, por exemplo, à formação do plural, como vimos nos dados do murle, em (4). Adicionalmente ao murle, podemos mencionar a redução de segmentos em truncamentos de empréstimos de compostos no japonês, fenômeno que se associa, consideravelmente, aos blends das línguas românicas. Nesses dados, a redução de segmentos é fruto de uma restrição fonológica imposta pela gramática da língua.

Nishihara, van de Weijer e Nanjo (2001) mostram que o apagamento de segmentos fonológicos no japonês visa adequar o empréstimo de uma palavra composta a uma forma com duas unidades bimoraicas $(2+2)$, sem gerar qualquer efeito estilístico, já que a estrutura prosódica básica do japonês é bimoraica. Exemplos desses dados são apresentados em (10).

5 Um(a) parecerista anônimo comentou que há uma diferença entre os truncamentos de palavras simples e de palavras compostas, uma vez que esses últimos parecem estar associados a uma adaptação fonológica, a qual visa reduzir seu número de palavras prosódicas, de duas (e.g., curta-metragem) para uma (e.g., curta). Entretanto, truncamento de compostos não é muito comum no português, e ocorre, em maior quantidade, nos compostos neoclássicos formados por uma combinação de radicais (e.g., morfo de morfologia, psico de psicologia, etc.). Esses compostos formam uma única palavra prosódica (cf. VIGÁRIO, 2003; NÓBREGA, 2014), e, portanto, colocam um sério problema para a hipótese de que truncamento de compostos decorre de uma redução no número de palavras prosódicas, tal como sugerido pelo(a) parecerista. 
(10)
a. hebii metaru
$\rightarrow$ hebi-meta de heavy metal 'heavy metal'
b. rajio kassetto rekoodaa $\rightarrow$ raji-kase de radio cassette recorder 'rádio cassete'
c. paasonaru konpyuutaa $\rightarrow$ paso-kon de personal computer 'laptop'
d. pantii sutokkingu $\rightarrow$ pan-suto de panty stockings 'meia calça'

(ITÔ, 1990, p. 220 apud NISHIHARA; van de WEIJER; NANJO, 2001, p. 300)

Assim sendo, os dados do japonês, bem como os dados do murle, evidenciam que a redução de segmentos e a morfologia não-concatenativa nem sempre estão associadas a um efeito estilístico, tal como ocorre nas línguas românicas. Portanto, embora verifiquemos o mesmo fenômeno superficialmente, as motivações que estão em jogo para a produção dessas palavras na gramática são distintas, ou seja, o que está em jogo no japonês e no murle, de um lado, e nas línguas românicas, de outro, são inputs distintos para a subtração de segmentos.

Nesses três casos de subtração, podemos identificar os seguintes inputs: no japonês, o que temos é um efeito de PF, exclusivamente, já que os empréstimos precisam ser adaptados à gramática fonológica da língua. No murle, observamos um caso de preenchimento ou não do nó funcional relativo à informação de número no componente morfológico. Já nos blends, o input para redução de segmentos está associado à emergência de efeitos estilísticos, como demonstraremos mais adiante. Essa variedade de motivações é uma característica comum da morfologia não-concatenativa - principalmente, se levarmos em consideração os dados discutidos na seção 2 -, a qual recebe esse rótulo apenas pelas semelhanças superficiais atestadas nos dados.

Parece-nos evidente, portanto, que a multiplicidade dos processos não-concatenativos presente nas línguas naturais exige uma explicação diversificada. Isso significa dizer que a não-concatenatividade morfológica não é uniforme na gramática e nem sempre decorre das mesmas motivações para o seu desencadeamento. Por isso, a depender do tipo de morfologia não-concatenativa com que estamos lidando, teremos de oferecer explicações distintas, nas quais podem estar em jogo não apenas a determinação do componente da gramática em que o processo ocorre, bem como o input imediato para sua atuação. Os blends, em particular, ilustram muito bem essa variabilidade, e serão discutidos mais atentamente na próxima seção.

\section{OS BLENDS E SUAS CARACTERÍSTICAS FONOLÓGICAS}

o blending é geralmente descrito como um processo de formação de palavras que ocorre através da fusão estrutural de duas palavras-fontes. Nos principais trabalhos que descreveram o processo, a característica mais 
explorada é a de seus aspectos fonológicos, o que é influenciado, em grande medida, pela sobreposição ou apagamento de segmentos idênticos (e.g., no $\mathrm{PB}$, apertamento < ap (a)rtamento + ape (rtado)). Há uma gama de trabalhos na literatura que ressalta tais similaridades fonológicas entre suas palavras-fontes, e, por esse motivo, é que comumente se postula que o input imediato para a formação de um blend é fonológico (cf. KUBOZONO, 1990; BAT-EL, 1996; PIÑEROS, 2000, 2004; GONÇALVES, 2006).

Também é recorrente na literatura a comparação entre o blending e a composição em termos fonológicos. Segundo Gonçalves (2003a), os blends diferem dos compostos por apresentar uma intersecção entre suas palavras-fontes, o que resulta em fusão e perda de material fônico não justificável por processos fonológicos segmentais. A composição, por outro lado, não altera a ordem linear de seus membros constituintes, de modo que não há uma inversão ou sobreposição dos segmentos, mesmo quando um determinado segmento é apagado por crase (e.g., aguardente < água + ardente), elisão (e.g., planalto $<$ plano + alto) ou haplologia (e.g., dedurar $<$ dedo + duro) - sendo esses últimos, processos fonológicos segmentais passíveis de regra ${ }^{6}$.

Entretanto, a diferença crucial entre esses dois processos de formação de palavras, defendida por Piñeros (2000) e Gonçalves (2003a, 2004b), diz respeito à formação da palavra prosódica. Enquanto nos compostos formados por duas palavras -, cada um dos membros projeta uma palavra prosódica (Pwd), gerando uma palavra prosódica complexa, tal como em (11a), nos blends, as duas palavras-fontes formam apenas uma Pwd, como em (11b):

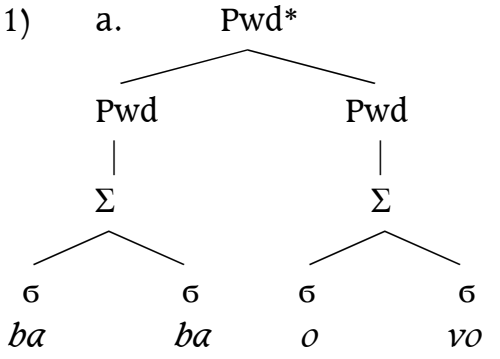

b.

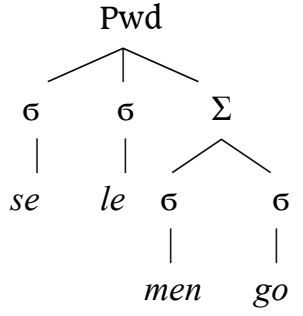

(GONÇALVES, 2003a, p.154)

Os blends, no entanto, não se restringem a formações que tenham a extensão de uma única palavra prosódica. No PB, por exemplo, encontramos blends formados a partir de duas palavras prosódicas, tal como dilma- 
lándra < Dílma + malandra, camisinholéncia < camisínha + violência Nèymaradóna < Neymár + Maradóna, entre outros. Sendo assim, a hipótese de que o blending parte de uma motivação fonológica, que visa reduzir o número de palavras prosódicas, não se verifica empiricamente, visto que esses últimos dados deveriam ser barrados pela gramática. Essas observações dificultam a assunção de que blends se comportam como verdadeiros compostos. Por esse motivo, assumimos que os blends são estruturas sintagmáticas encurtadas, na linha dos autores mencionados acima, porém com diferentes formas de externalização, as quais serão detalhadas na seção 5 .

Em contrapartida, Minussi e Nóbrega (2014) argumentam que o input para a criação dos blends é semântico, uma vez que a ausência de segmentos idênticos em blends como boacumba < boa + macumba, showmício < show + comício e acãoxonado < apaixonado + cão não bloqueia sua formação. Além disso, o caráter cômico/jocoso associado aos blends deriva, primeiramente, da oposição semântica entre suas palavras-fontes (e.g., boilarina < boi + bailarina), ou da modificação causada por uma das palavras-fontes à outra (e.g., apertamento < apartamento + apertado), mas não da sobreposição ou apagamento de segmentos fonológicos.

Por exemplo, não encontramos a mesclagem de duas palavras sem qualquer relação semântica entre si que possibilite um efeito cômico ou jocoso. De fato, o que há de mais saliente na formação dos blends é o apagamento ou sobreposição de segmentos, porém, isso parece ocorrer somente quando há uma motivação semântica para a mesclagem, a qual viabiliza os efeitos estilísticos encontrados. Tal é o fato de que nem todos os blends ocorrem com sobreposição ou apagamento de segmentos fonológicos, como evidenciamos acima.

Essa variedade interna entre os blends exige uma divisão dos produtos formados por esse processo de formação de palavras. A partir da caracterização inicial sugerida por Basilio (2005) e Gonçalves e Almeida (2007), trazemos a classificação dos blends adaptada por Minussi e Nóbrega (2014). Com base nessa divisão, a formação dos blends distribui-se por três categorias gerais de acordo com critérios morfofonológicos e semânticos, conforme descrito na Tabela 1, abaixo: 
Tabela 1 - Classificação dos blends (MINUSSI; NÓBREGA, 2014, p. 168)

\begin{tabular}{|c|c|c|}
\hline Blends Fonológicos & Blends Morfológicos & Blends Semânticos \\
\hline $\begin{array}{l}\text { - presença de um ou } \\
\text { mais segmentos fono- } \\
\text { lógicos idênticos sobre- } \\
\text { postos. }\end{array}$ & $\begin{array}{l}\text { - ausência de quaisquer } \\
\text { segmentos fonológicos } \\
\text { idênticos nas palavras- } \\
\text {-fontes; } \\
\text {-truncamento de uma } \\
\text { ou de ambas as pala- } \\
\text { vras fontes. }\end{array}$ & $\begin{array}{l}\text { - reanálise semânti- } \\
\text { ca de uma parte dos } \\
\text { segmentos fonológicos } \\
\text { de uma das palavras- } \\
\text {-fontes; } \\
\text { - substituição do seg- } \\
\text { mento fonológico rea- } \\
\text { nalisado por segmentos } \\
\text { fonológicos equivalen- } \\
\text { tes ao de outra raiz com } \\
\text { traços enciclopédicos } \\
\text { distintos. }\end{array}$ \\
\hline $\begin{array}{l}\text { Por exemplo: } \\
\text { - matel < mato }+ \text { motel } \\
\text { - roubodízio < roubo }+ \\
\text { rodízio }\end{array}$ & $\begin{array}{l}\text { Por exemplo: } \\
\text { - cariúcho < carioca }+ \\
\text { gaúcho } \\
\text { - portunhol < portu- } \\
\text { guês }+ \text { espanhol }\end{array}$ & $\begin{array}{l}\text { Por exemplo: } \\
\text { - boacumba < }(\text { má)- } \\
\text {-cumba } \\
\text { - bebemorar < (come)- } \\
\text {-morar }\end{array}$ \\
\hline
\end{tabular}

Na próxima seção, apresentamos o modelo da MD, juntamente com as adaptações propostas por Minussi e Nóbrega (2014) para uma análise eficiente dos processos não-concatenativos que desencadeiam efeitos estilísticos, tal como os blends.

\section{A Morfologia Distribuída e o tratamento dos bLENDS}

A MD é uma abordagem realizacional não-lexicalista para a gramática, na qual a interface entre a sintaxe e a morfologia procura ser a mais transparente possível, tendo em vista que, nessa abordagem, as mesmas operações responsáveis pela formação de sentenças (i.e., Merge, Move, Copy) são também responsáveis pela formação da estrutura interna das palavras. Assim sendo, tanto palavras quanto sentenças são geradas em um mesmo ambiente computacional, a sintaxe.

Nessa visão de gramática, o que era tipicamente assumido como informação presente no léxico foi distribuído pela arquitetura da gramática através de três listas. A Lista 1, que contém os primitivos que alimentam a sintaxe, ou seja, raízes $(\sqrt{ })$ e morfemas abstratos, os quais são constituídos 
de feixes de traços gramaticais (e.g., [n], [v], [plural], [passado]). A Lista 2 contém os itens de vocabulário (IVs), ou seja, conexões entre um conjunto de traços gramaticais e seus respectivos traços fonológicos, os quais são inseridos pós-sintaticamente, ou seja, após a derivação da estrutura sintática da palavra. E, finalmente, a Lista 3, que contém as entradas enciclopédicas que relacionam IVs a significados.

Tendo em mente os pressupostos dessa teoria, o principal desafio que os processos não-concatenativos suscitam é o de como explicar a emergência dos efeitos estilísticos presentes em seus dados sem assumir um léxico gerativo. Como uma alternativa de análise, sugerimos que a Lista 3 detém um papel fundamental no desencadeamento de marcas estilísticas nos processos de formação de palavras, ao influenciar as operações do componente morfológico. Assumimos que uma interface entre PF e LF pode se dar via Lista 3, no momento em que essa última acessa a interface conceitual. Esse acesso, por sua vez, garante uma verificação do que foi criado pela fonologia e aquilo que é interpretado por LF.

Nesse sentido, a Lista 3 deve ser vista como um filtro. Nela, existem informações concernentes ao nosso conhecimento de mundo, que viabilizam a interpretação de uma raiz em determinado contexto (e.g., VGATO: (i) mamífero, (ii) homem bonito; (iii) ligação clandestina de fios, etc.), bem como a interpretação de construções sintáticas (e.g., expressões idiomáticas), e a adequação prosódica da estrutura fonológica com determinado conteúdo semântico. Consequentemente, a Lista 3 deve acessar o componente morfológico, como indica a seta saindo da Lista 3 para o Vocabulário, na Figura 1 , a fim de desencadear tais efeitos pragmáticos.

Figura 1 - Arquitetura da gramática para MD adaptada por Minussi e Nóbrega (2014, p. 172)

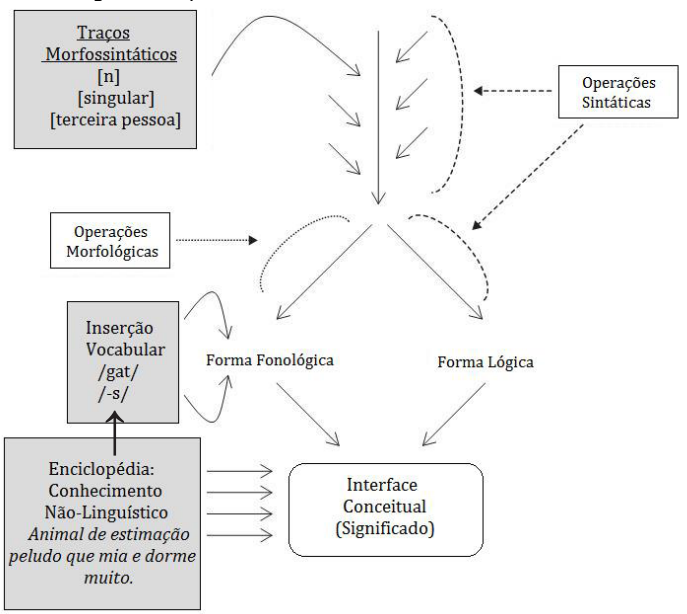

Revista Letras, Curitiba, N. 91 P. 158-177, JAN./JUN. 2015. ISSN 2236-0999 (VERSÃo ELETRÔNICA) 
Há, entretanto, outras propostas não-lexicalistas que tentam explicar os efeitos estilísticos provenientes de processos não-concatenativos. Uma dessas propostas é a de Scher (2013) que, ao analisar as formas truncadas do PB, assume um morfema avaliador [Eval] presente na estrutura sintática da palavra, o qual é responsável por promover a leitura apreciativa de seus dados. Um problema para essa proposta é a ausência de uma motivação justificada para a assunção de que a sintaxe opera com informações dessa natureza. Acreditamos que a morfologia não-concatenativa do PB deve ser analisada pós-sintaticamente, uma vez que a sintaxe, a priori, não está preocupada em criar tais efeitos.

\section{UMA ANÁLISE PÓS-SINTÁTICA COM BASE NA ENCICLOPÉDIA}

Nesta seção, recuperamos a análise de Minussi e Nóbrega (2014), que propõem uma análise pós-sintática para os blends com base no acesso da Enciclopédia durante a inserção de vocabulário. Como já foi mencionado, os autores assumem que o input imediato para a formação dos blends não é fonológico, ou seja, a mesclagem não é motivada por uma semelhança entre os segmentos das palavras-fontes, mas é semântico, motivado por uma checagem dos traços enciclopédicos que compõem essas palavras.

Os autores distinguem a formação dos blends fonológicos e semânticos da formação dos blends morfológicos. Os primeiros são formados a partir de duas raízes categorizadas, como em um sintagma, enquanto os blends morfológicos são formados por uma única raiz, de modo que sua formação ocorre como uma palavra derivada, em que há uma raiz e um afixo. Neste artigo, expandimos a proposta dos autores para os blends fonológicos, a fim de mostrar sua eficácia.

Encontramos em Minussi e Nóbrega (2014) um passo-a-passo da derivação dos blends, o qual reproduzimos abaixo:

(i) O primeiro passo é a combinação dos primitivos fornecidos pela Lista 1 - raízes e morfemas abstratos - através da operação Merge;

(ii) Em seguida, há o envio da estrutura sintática para as interfaces fonológica (PF) e semântica (LF);

(iii) No caminho para PF, onde se localiza o componente morfológico, serão inseridos os IVs nos nós terminais gerados pela sintaxe, por meio de uma operação de inserção de vocabulário. 
Logo após a linearização da estrutura sintática e inserção de vocabulário, ocorre o acesso da Lista 3, segundo os autores. Tal acesso é o responsável por desencadear a mesclagem, pois ele licencia o apagamento de material fonológico, criando os efeitos estilísticos encontrados nos blends. Desse modo, o apagamento ocorrerá de acordo com a quantidade de segmentos fonológicos idênticos presentes nas palavras-fontes, a partir dos seguintes critérios:

(i) Se há similaridades fonológicas entre os dois elementos constituintes e esses elementos são formados por duas raízes, as palavras-fontes sofrerão uma sobreposição e, como resultado, teremos um blend fonológico.

(ii) Se não houver segmentos fonológicos idênticos, as palavras-fontes deverão se sobrepor nos pontos em que há um alto grau de similaridade suprassegmental, por exemplo, sílaba acentuada ${ }^{7}$ , resultando em um blend morfológico.

(iii) Nos casos de blends semânticos, há uma reanálise de segmentos de uma das palavras-fontes. Isso acontece quando o acesso da Lista 3 ao resultado da inserção de vocabulário reinterpreta segmentos fonológicos como uma raiz, e causa a substituição dos segmentos reanalisados por segmentos equivalentes a outra raiz com traços enciclopédicos opostos.

(MINUSSI; NÓBREGA, 2014, p. 177)

Segundo os autores, os traços enciclopédicos diferenciam-se dos traços semânticos. Os traços semânticos que podem ser relevantes para sintaxe são definitude, animacidade, telicidade etc. Os traços enciclopédicos são aqueles contidos nas raízes e correspondem às informações relacionadas ao nosso conhecimento de mundo. Se tomarmos uma raiz como $\sqrt{ }$ CACHORR- e listarmos seus traços principais, por exemplo, canino, mamífero, com pelos, que late etc., não poderemos dizer que essas informações são equivalentes a traços semânticos como definitude, animacidade, contável etc., mas são informações derivadas de nosso conhecimento de mundo com relação a esse referente.

Assim sendo, Minussi e Nóbrega (2014) demonstram as etapas da formação dos blends através dos seguintes esquemas:

7 Ver Gonçalves (2003a), para casos como portunhol < português + espanhol, espaguês $<$ espanhol + inglês e cariúcho < carioca + gaúcho. 
(12)

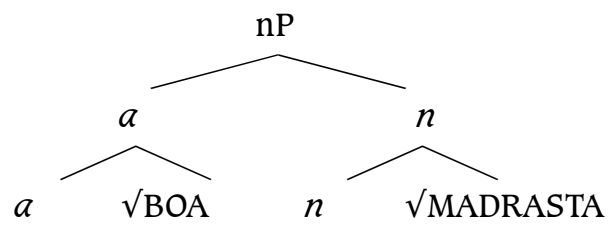

(13)

/boua/

/ma.dras.ta/

[-Dinâmico, + escalar [+com, abst, +an] $([\mathrm{]}])$

$[$ coisa [tcom,-abst, + an] $[$ [ ] $])]$

$<$ qualidade $>$

$<$ mulher do meu pai>

$<$ virtude $>$

$<$ não é a mãe biológica >

$<$ retidão $>$

$<$ mulher ruim $>$

$<$ incapaz de expressar afeto $>$

$<$ não é protetora $>$

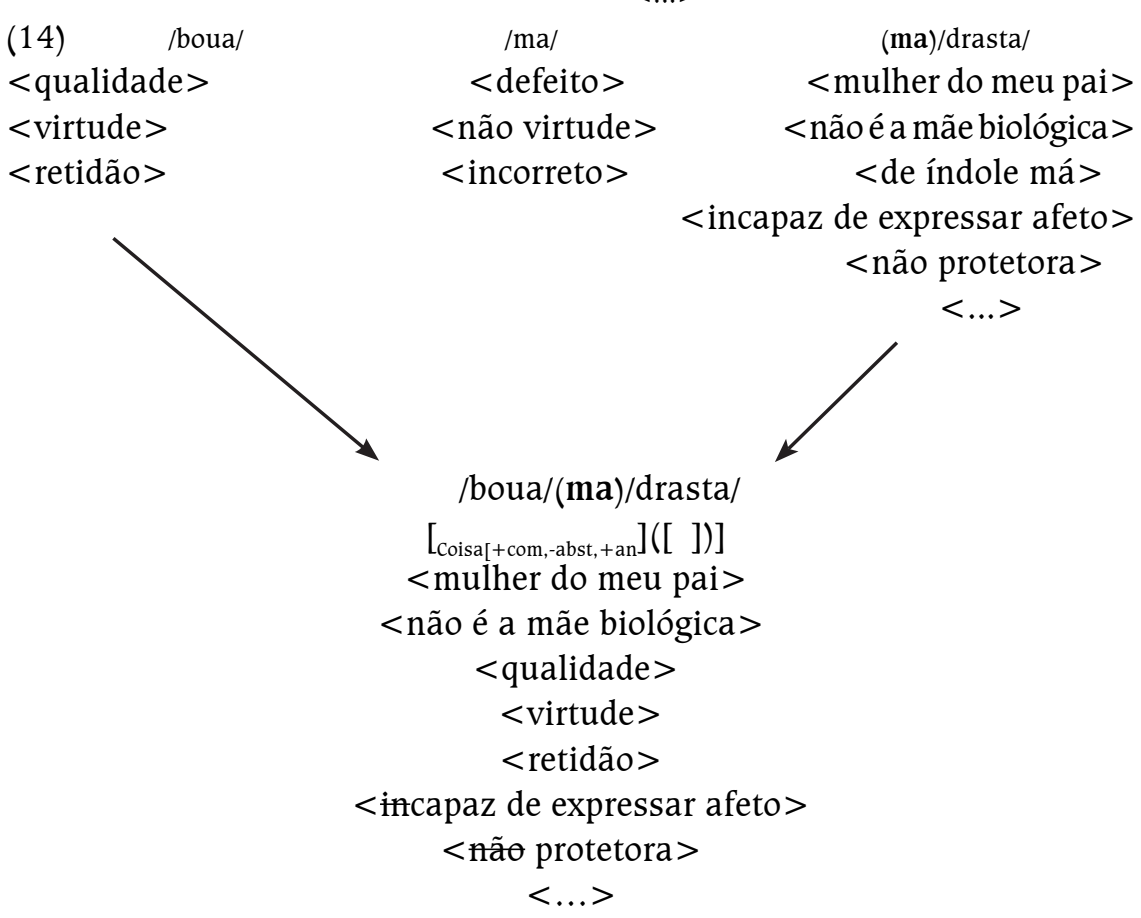

O mesmo cálculo ocorrerá em blends como bebemorar, boacumba, acãoxonados, em que os segmentos sublinhados são substituídos devido à análise enciclopédica. Na próxima seção, descrevemos a derivação dos blends fonológicos. 
5. 1. CoRROBORANDO A ANÁLISE: OS BLENDS FONOLÓGICOS

Tomemos a estrutura sintática do blend fonológico namorido $<$ namorado + marido $>$, representada em (15).

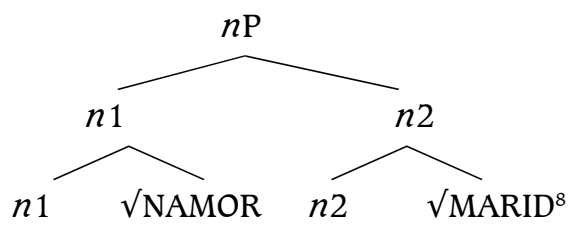

Uma vez formada na sintaxe, a estrutura em (15) é enviada para PF e LF após o Spell-Out sintático. No caminho para PF, serão inseridos os IVs em seus nós terminais. Como já dissemos, é nesse ponto da derivação, após a inserção de vocabulário e linearização da estrutura sintática, que ocorre o acesso da Lista 3. Tal acesso serve de input para a mesclagem de $n 1$ e $n 2$. Com o acesso da Lista 3, dá-se a checagem dos traços enciclopédicos para a formação do blend namorido, que se processa, idealmente, como no esquema abaixo:

$$
\begin{aligned}
& \text { namorado } \\
& \text { [Coisa [+com, -abst, }+ \text { an] }[(\mathrm{i}])] \\
& \text { <não-casado> } \\
& <\text { não mora junto }> \\
& <\text { apaixonado> } \\
& <\text { companheiro }> \\
& <\ldots>
\end{aligned}
$$

marido

$$
\begin{gathered}
{[\text { [coisa, [+com,-abst, }+ \text { na] }[(i])]} \\
<\text { casado }> \\
<\text { mora junto }> \\
<\text { apaixonado }> \\
<\text { companheiro }> \\
<\ldots>>
\end{gathered}
$$

Em (16), namorado e marido possuem traços enciclopédicos semelhantes (e.g., apaixonado e companheiro) e traços opostos (e.g., não-casado vs. casado, mora junto vs. não-mora junto). Essa oposição de traços produz o efeito estilístico presente nesse e em outros blends, desencadeando a mesclagem. Após a intervenção da Lista 3, e devido à presença de segmentos idênticos nas duas palavras-fontes, temos uma sobreposição de segmentos, reduzindo a extensão da palavra. No caso do blend namorido, a sobreposição ocorre nos seguintes segmentos: /m/, /r/ e /do/, como podemos observar em (17):

8 Na estrutura em (15), os expoentes fonológicos do categorizador nominal $n 1$ é /-ad-/, e as vogais finais /o/ dizem respeito às marcas de classe nominal, inseridas pós-sintaticamente. 
(17)

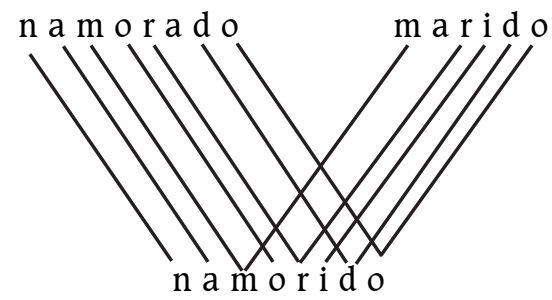

Essa análise aproxima-se de trabalhos de cunho fonológico, cujas soluções apontam que o input imediato para a mesclagem decorre de pressões fonológicas (cf. GONÇALVES, 2003a, 2003b, 2004b, 2006). Entretanto, não há nada na estrutura dessas palavras que desencadeie tal sobreposição, visto que elas poderiam muito bem ser empregadas integralmente (e.g., em um composto como namorado-marido, ou cantora-atriz, do blend cantriz). Defendemos, portanto, que é a Lista 3 a responsável por desencadear a sobreposição de segmentos, ao mapear traços enciclopédicos opostos em dois nós complexos (i.e., duas raízes categorizadas), permitindo, assim, a formação de um blend.

\section{Considerações Finais}

Neste artigo, apresentamos uma análise para os blends fonológicos, corroborando a análise de Minussi e Nóbrega (2014). Acreditamos que os traços semânticos relevantes para a sintaxe são outros, e os traços contidos na raiz são de ordem enciclopédica, já que correspondem a informações concernentes ao nosso conhecimento de mundo. Demonstramos que a morfologia não-concatenativa é um rótulo amplo que abriga uma série de fenômenos morfológicos, os quais ocorrem em diferentes pontos da gramática a partir de diferentes inputs.

Por fim, reiteramos que o input imediato para a formação de um blend advém de fatores semânticos, e, de acordo com as ferramentas fornecidas pela MD, isso decorre de um acesso da Lista 3 ao componente morfológico da gramática. Com base nesse sistema, admitimos que a sobreposição ou apagamento de segmentos é um epifenômeno e não uma exigência imprescindível para a formação de um blend. 


\section{REFERÊNCIAS}

ARAD, M. Roots and Patterns: Hebrew Morpho-syntax. Studies in Natural Language and Linguistic Theory. Amsterdam: Sprinter, 2005.

ARENSEN, J. Murle Grammar. Juba: SIL Sudan, 1982.

BASILIO, M. A Fusão Vocabular como Processo de Formação de Palavras. Trabalho apresentado no 4. Congresso Internacional da ABRALIN, 2005.

BAT-EL, O. Selecting the best of the worst: the grammar of Hebrew blends. Phonology and Linguistic Theory, v. 12, p. 571-596, 1996.

.In search for the roots of the $C$-root: The essence of Semitic morphology. The Workshop on Roots and Template Morphology. Los Angeles: USC, 2001. Disponível em <http://www. outibatel.com/wp-content/uploads/2010/12/Bat-El-2001-C-root.pdf>. Acesso em: 10 ago. 2009.

BYE, P.; SVENONIUS, P. Non-concatenative morphology as epiphenomenon. In: TROMMER, J. (ed.). The Morphology and Phonology of Exponence. Oxford University Press, 2012. p. 427-495.

DAVIS, S.; TSUJIMURA, N. Non-concatenative Derivation: Other Processes. In: LIEBER, R.; STEKAUER, P. (eds.) The Oxford Handbook of Derivational Morphology. Oxford: Oxford University Press, 2014. p. 190-218.

DON, J. On conversion, Relisting and Zero-derivation: A comment on Rochelle Lieber - English word-formation processes. SKASE Journal of Theoretical Linguistics, v.2, n.2, p. 2-16, 2005.

EMBICK, D. Localism vs Globalism in Morphology and Phonology. Cambridge, Mass.: MIT Press, 2010.

GONÇALVES, C. A. V. A ambimorfemia de cruzamentos vocabulares: uma abordagem por ranking de restrições. Revista da Abralin, v. 5, n.1, p. 169-183, 2006.

A morfologia prosódica e o comportamento transderivacional da hipocorização no português brasileiro. Revista de Estudos da Linguagem, v. 12, n. 1, p. 7-32, 2004a.

. Blends lexicais em português: não-concatenatividade e correspondência. Veredas, v.7, n.1, p.149-167, 2003a.

. Cruzamento Vocabular em Português: A questão das fronteiras com outros processos de criação de palavras. CONGRESSO NACIONAL DA ABRALIN, 3., 2003, Niterói. Anais... Niterói: UFF - Centro de Estudos Gerais, v. 1, 2003b. p. 824-831.

. Processos morfológicos não-concatenativos do português brasileiro: formato morfopro-

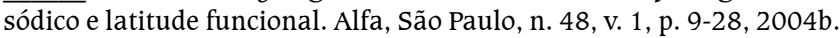
. Uma abordagem autossegmental para a morfologia. Cadernos de Letras da UFF - Dossiê: Difusão da língua portuguesa, n. 39, p. 211-232, 2009.

GONÇALVES, C. A. V.; ALMEIDA, M. L. L. Bases semântico-cognitivas para a diferenciação de cruzamentos vocabulares. Revista Portuguesa de Humanidades, v. 11, p. 75-85, 2007.

HALLE, M.; MARANTZ, A. Distributed Morphology and the Pieces of Inflection. In: HALE, K.; KEYSER, S. K. (orgs.) The View from Building 20; Essays in Honor of Sylvain Bromberger. Cambridge, Mass: MIT Press, 1993. p. 111-176.

HASPELMATH, M.; SIMS, A. D. Understanding Morphology. 2. ed. Londres: An Hachette UK Company, 2010.

ITÔ, J. Prosodic minimality in Japanese. In: ZIOLKOWSKI, M. et al (orgs.) CLS, v. 26, n. 2. Chicago: Chicago Linguistic Society, 1990. p. 213-239. 
KIPARSKY, P. Word-formation and the Lexicon. In: INGEMANN, F. (ed.) Proceedings of the Mid-America Linguistics Conference. Lawrence: Kansas, 1982.

KUBOZONO, H. Phonological constraints on blending in English as case for phonology -morphology interface. Yearbook of Morphology, v. 3, p. 1-20, 1990.

KURISU, K. The Phonology of Morpheme Realization. Thesis (PhD) - University of California, Santa Cruz. ROA 490-0102, 2001.

MARANTZ, A. Re Reduplication. Linguistic Inquiry, v. 13, n. 3, p. 435-482, 1982.

. No Escape from Syntax: Don't try Morphological Analysis in the privacy of your own Lexicon. In: DIMITRIADIS, L. S.; SUREK-CLARK, C.; WILLIAMS, A. (orgs.) Proceedings of the 21st Penn Linguistics Colloquium, v. 4, n. 2, p. 201-225, 1997.

MINUSSI, R. D. Os sabores do nome: um estudo sobre a seleção de argumentos e as nominalizações do hebraico. Tese (Doutorado em Letras) - Faculdade de Filosofia, Letras e Ciências Humanas, Universidade de São Paulo. São Paulo, 2012.

MINUSSI, R. D.; NÓBREGA, V. A interface sintaxe-pragmática na formação de palavras: avaliando os pontos de acesso da Enciclopédia na arquitetura da gramática. Veredas (UFJF), v.18, n. $1,2014$.

NISHIHARA, T; van de WEIJER, J.; NANJO, K. Against headedness in compound truncation: English compounds in Japanese. In: van de WEIJER, J.; NISHIHARA, T. (orgs.) Issues in Japanese phonology and morphology. Berlim: Mouton de Gruyter, p. 299-324, 2001.

NÓBREGA, V. A. Tópicos em composição: estrutura, formação e acento. Dissertação (Mestrado em Letras) - Faculdade de Filosofia, Letras e Ciências Humanas, Universidade de São Paulo. São Paulo, 2014.

PIÑEROS, C.-E. Word-blending as a case of non-concatenative morphology in Spanish. Rutgers Optimality Archive, 2000.

.The creation of portmanteaus in the extragrammatical morphology of Spanish. Probus, v. 16, p. 203-240, 2004.

SANDMANN, A. J. Morfologia Lexical. São Paulo: Contexto, 1990.

SCHER, A. P. Formas truncadas em português brasileiro e espanhol peninsular: descrição preliminar. ReVEL, ed. esp. n. 5, p. 61-79, 2011.

Concatenative affixation in Brazilian Portuguese truncated forms. In: Proceedings of the Glow in Asia IX 2012: the main session. Tsu: Mie University, 2013. p. 261-270.

SIDDIQI, D. Syntax within the Word: economy, allomorphy, and the argument selection in Distributed Morphology. Amsterdam: John Benjamins Publishing Company, 2009.

VAZQUEZ, R. P. A criação lexical via formas truncadas: uma análise do fenômeno no espanhol. Rio de Janeiro: Faculdade de Letras/UFRJ, 2008.

VILlALVA, A. Morfologia do Português. Lisboa: Universidade Aberta, 2008.

Submetido em: 02/03/2015

Aceito em: 22/05/2015 